\title{
Doppler Synthetic Aperture Radar Imaging
}

\author{
Ling Wang ${ }^{a}$ and Birsen Yazıc1 ${ }^{b}$ \\ ${ }^{a}$ Department of Information and Communication Engineering, Nanjing University of \\ Aeronautics and Astronautics, Nanjing, Jiangsu 210016 China; \\ ${ }^{b}$ Department of Electrical, Computer and System Engineering, Rensselaer Polytechnic \\ Institute, Troy, NY 12180 USA
}

\begin{abstract}
We consider synthetic aperture radar system using ultra-narrowband continuous waveforms, which we refer to as Doppler Synthetic Aperture Radar (DSAR). We present a novel image formation method for bi-static DSAR. Our method first correlates the received signal with a scaled or frequency-shifted version of the transmitted signal over a finite time window, and then uses microlocal analysis to reconstruct the scene by a filtered-backprojection of the correlated signals. Our approach can be used under non-ideal imaging scenarios such as arbitrary flight trajectories and non-flat topography. Furthermore, it is an analytic reconstruction technique which can be made computationally efficient. We present numerical experiments to demonstrate the performance of the proposed method.
\end{abstract}

Keywords: Imaging, Synthetic Aperture, Continuous Wave (CW), Doppler, Backprojection

\section{INTRODUCTION}

We consider a synthetic aperture radar imaging system where the transmitter transmits ultra-narrowband or single-frequency continuous waveforms. Due to the high-Doppler-resolution nature of the transmitted waveforms, we refer to the system under consideration as Doppler synthetic aperture radar (DSAR).

As compared to the traditional SAR system where wideband waveforms are used to obtain high range resolution, DSAR requires a relatively simple and low-cost transmitter. It does not even need a dedicated transmitter when existing electronic signals, such as radio and television signals and cell phone signals are used as the illumination sources of DSAR. These waveforms are continuous, ultra-narrowband, and have high Doppler resolution.

Radar imaging with ultra-narrowband waveforms has received interest before [1-9]. In [1,2], the imaging of rotating objects using a single-frequency continuous wave $(\mathrm{CW})$ illumination was investigated theoretically. The same approach was recently studied experimentally in [3]. In [4,5], the imaging of stationary objects using ultra-narrowband or narrowband CW with spatially distributed radars was studied. All of these studies use tomographic image formation methods that rely on trading the resolution achieved by bandwidth with the resolution achieved by spatial diversity.

An alternative approach to ultra-narrowband radar imaging relies on the idea of imaging scatterers that lie on iso-Doppler curves [6-9]. Our work falls into this category. This idea was first introduced in [7]. In [8], the idea of imaging scatterers lying on iso-Doppler contours was introduced to map polar regions of terrestrial planets using the Doppler shifts of the received signal. The data was collected for several repeated passes over the polar region. A filtered-backprojection (FBP) type inversion formula was presented to recover the surface reflectivity from its projections onto weighted iso-Doppler contours. In [6], the mono-static synthetic aperture imaging using high-Doppler-resolution measurements was discussed. The same idea introduced in $[7,8]$ was explored. An expedient, approximate implementation of the imaging method based on Radon-Hough transform relying on detecting the lines associated with the scatterers was presented. Both the resolution analysis and the

Further author information: (Send correspondence to B.Y.)

B.Y.: E-mail: yazici@ecse.rpi.edu, Telephone: +1(518)276 2905, Fax: +1(518)276 6261

L.W.:E-mail: wanglrpi@gmail.com

Algorithms for Synthetic Aperture Radar Imagery XVIII, edited by Edmund G. Zelnio, Frederick D. Garber, Proc. of SPIE Vol. 8051, 80510F · @ 2011 SPIE · CCC code: 0277-786X/11/\$18 · doi: 10.1117/12.883850 
imaging method presented in [6] are limited to the straight flight path and flat topography. In [9], we explored the idea of imaging scatterers on iso-Doppler curves for passive synthetic aperture imaging using ultra-narrowband waveforms. We introduced a concept of passive Doppler-scale factor and corresponding passive iso-Doppler curves. The image reconstruction was performed via an FBP-type inversion. This work is particularly suitable for non-cooperative transmitters of opportunity where the locations of the transmitting antennas are unknown.

We present a novel filtered-backprojection (FBP) type image formation method for bi-static DSAR. Our method first correlates the received signal with a scaled or frequency-shifted version of the transmitted signal over a finite time window. The high-frequency analysis of the resulting model shows that the correlated received signal is the projections of the scene onto the bi-static iso-Doppler curves. We use microlocal techniques to develop a FBP reconstruction of the scene. We present numerical experiments to demonstrate the performance of the image reconstruction method.

Our method is applicable to arbitrary, but known flight trajectories and non-flat topography. It doesn't require antenna with high directivity and can be used with stationary and/or mobile transmitters. Furthermore, it is an analytic reconstruction technique which can be made computationally efficient.

The organization of the paper is as follows. In Section 2, we present the model for the received signal and develop the forward model for bistatic DSAR. In Section 3, we develop an FBP-type image formation method for bistatic DSAR.In Section 4, we present numerical simulations to demonstrate the performance of the proposed DSAR imaging method. Section 5 concludes our paper.

We use the following notational conventions throughout the paper. The bold Roman, bold italic and Roman lower-case letters are used to denote variables in $\mathbb{R}^{3}, \mathbb{R}^{2}$ and $\mathbb{R}$, respectively, i.e., $\mathbf{z}=(\boldsymbol{z}, z) \in \mathbb{R}^{3}$, with $\boldsymbol{z} \in \mathbb{R}^{2}$ and $z \in \mathbb{R}$. The calligraphic letters $(\mathcal{F}, \mathcal{K}$ etc.) are used to denote operators.

\section{FORWARD MODEL FOR IMAGING}

Let the earth's surface denoted by $\mathbf{z}=(\boldsymbol{z}, \boldsymbol{\psi}(\boldsymbol{z})) \in \mathbb{R}^{3}$, where $\boldsymbol{z} \in \mathbb{R}^{2}$ and $\boldsymbol{\psi}: \mathbb{R}^{2} \rightarrow \mathbb{R}$ is a known function for the ground topography. Furthermore, we assume that the scattering takes place in a thin region near the surface. Thus, the reflectivity function has the form

$$
V(\mathbf{z})=\rho(\boldsymbol{z}) \delta(z-\boldsymbol{\psi}(\boldsymbol{z}))
$$

We consider a bistatic SAR (BISAR) imaging system. Let $\gamma_{T}(t)$ and $\gamma_{R}(t)$ be the transmitter and receiver trajectories, respectively, and let $s(t)$ denote the received signal of the receiver. Thus, the received signal for BISAR with transmitter transmitting an ultranarrow-band CW waveform can be expressed as

$$
s(t)=\int \mathrm{e}^{\mathrm{i} \omega_{0}\left(t-R_{T R}(t, \boldsymbol{z}) / c_{0}\right)} \tilde{p}\left(t-R_{T R}(t, \boldsymbol{z}) / c_{0}\right) A_{T R}\left(\omega_{0}, \mathbf{z}, \boldsymbol{\gamma}_{R}(t), \boldsymbol{\gamma}_{T}(t)\right) \rho(\boldsymbol{z}) d \boldsymbol{z}
$$

where $\omega_{0}$ denotes the carrier frequency and $\tilde{p}(t)$ is the complex envelope of the transmitted waveform $p(t)$, which is slow varying as a function of $t$ compared to $\mathrm{e}^{\mathrm{i} \omega_{0} t}$;

$$
R_{T R}(t, \boldsymbol{z})=\left|\gamma_{T}(t)-\mathbf{z}\right|+\left|\mathbf{z}-\gamma_{R}(t)\right|
$$

is the bistatic range, and $A_{T R}\left(\omega_{0}, \mathbf{z}, \gamma_{R}(t), \gamma_{T}(t)\right)$ is given by

$$
A_{T R}=\frac{\omega_{0}^{2}}{(4 \pi)^{2}\left|\gamma_{R}(t)-\mathbf{z}\right|\left|\mathbf{z}-\gamma_{T}(t) T\right|} J_{\mathrm{tr}}\left(\omega_{0}, \gamma_{R}(t), \gamma_{R}(t)\right) J_{\mathrm{rc}}\left(\omega_{0}, \mathbf{z}, \gamma_{T}(t)\right)
$$

where $J_{t r}$ and $J_{r c}$ are the transmitter and receiver antenna beam pattern related terms, respectively.

We define the correlation of the received signal given by (2) with a scaled or frequency-shifted version of the transmitted signal over a finite time window by

$$
d(\tau, \mu)=\int s(t+\tau) p^{*}(\mu t) \phi(t) d t
$$


for some $\tau \in \mathbb{R}$ and $\mu \in \mathbb{R}^{+}$, where $\phi(t)$ is a smooth windowing function centered at $t=0$ with finite support.

Substituting (2) into (5), we have

$$
d(\tau, \mu)=\int \mathrm{e}^{\mathrm{i} \omega_{0}\left(t+\tau-R_{T R}(t+\tau, \boldsymbol{z}) / c_{0}\right)} \mathrm{e}^{-\mathrm{i} \omega_{0} \mu t} A_{\tilde{p}}(\mathbf{z}, t, \tau, \mu) A_{T R}\left(\omega_{0}, \mathbf{z}, t, \tau\right) \rho(\boldsymbol{z}) d \boldsymbol{z} \phi(t) d t
$$

where $R_{T R}(t+\tau, \boldsymbol{z})$ is given by (3) with the time variable $t$ replaced with $t+\tau$,

$$
A_{\tilde{p}}(\mathbf{z}, t, \tau, \mu)=\tilde{p}\left(t+\tau-R_{T R}(t+\tau, \boldsymbol{z}) / c_{0}\right) \tilde{p}^{*}(\mu t)
$$

and

$$
A_{T R}\left(\omega_{0}, \mathbf{z}, t, \tau\right)=A_{T R}\left(\omega_{0}, \mathbf{z}, \gamma_{R}(t+\tau), \gamma_{T}(t+\tau)\right)
$$

Now using the Taylor expansion of $\gamma_{T}(t+\tau)$ and $\gamma_{R}(t+\tau)$ at $t=0$, we approximate

$$
\left|\gamma_{T}(t+\tau)-\mathbf{z}\right| \approx\left|\gamma_{T}(\tau)-\mathbf{z}+\dot{\gamma}_{T}(\tau) t\right| \approx\left|\gamma_{T}(\tau)-\mathbf{z}\right|+\widehat{\gamma_{T}(\tau)}-\mathbf{z} \cdot \dot{\gamma}_{T}(\tau) t
$$

and

$$
\left|\mathbf{z}-\gamma_{R}(t+\tau)\right| \approx\left|\mathbf{z}-\gamma_{R}(\tau)-\dot{\gamma}_{R}(\tau) t\right| \approx\left|\mathbf{z}-\gamma_{R}(\tau)\right|-\mathbf{z} \widehat{-\gamma_{R}}(\tau) \cdot \dot{\gamma}_{R}(\tau) t
$$

Note that $\mathbf{z} \widehat{-\gamma_{R}(\tau)}$ denotes the unit vector in the direction of $\mathbf{z}-\gamma_{R}(\tau)$.

Substituting (9) and (10) into (6), after rearranging the exponential terms, we obtain

$$
\begin{aligned}
d(\tau, \mu) \approx \int \mathrm{e}^{-\mathrm{i} \omega_{0} t\left[\mu-1+\left(\boldsymbol{\gamma}_{T} \widehat{(\tau)}-\mathbf{z} \cdot \dot{\gamma}_{T}(\tau)+\widehat{\boldsymbol{\gamma}_{R}(\tau)-} \mathbf{z} \cdot \dot{\gamma}_{R}(\tau)\right) / c_{0}\right]} \mathrm{e}^{\mathrm{i} \omega_{0}\left(\tau-R_{T R}(\tau, \boldsymbol{z}) / c_{0}\right)} \\
\times A_{\tilde{p}}(\mathbf{z}, t, \tau, \mu) A_{T R}\left(\omega_{0}, \mathbf{z}, t, \tau\right) \rho(\boldsymbol{z}) d \boldsymbol{z} \phi(t) d t
\end{aligned}
$$

We write the forward model for bistatic DSAR as follows:

$$
\begin{aligned}
d(\tau, \mu) & \approx \mathcal{F}[\rho](\tau, \mu) \\
& =\int \mathrm{e}^{-\mathrm{i} \varphi(t, \boldsymbol{z}, \tau, \mu)} A(\boldsymbol{z}, t, \tau, \mu) \rho(\boldsymbol{z}) d t d \boldsymbol{z}
\end{aligned}
$$

where

$$
\varphi(t, \boldsymbol{z}, \tau, \mu)=\omega_{0} t\left[\mu-1+\left(\widehat{\gamma_{T}(\tau)}-\mathbf{z} \cdot \dot{\gamma}_{T}(\tau)+\widehat{\gamma_{R}(\tau)}-\mathbf{z} \cdot \dot{\gamma}_{R}(\tau)\right) / c_{0}\right]
$$

and

$$
A(\boldsymbol{z}, t, \tau, \mu)=\mathrm{e}^{\mathrm{i} \omega_{0}\left(\tau-R_{T R}(\tau, \boldsymbol{z}) / c_{0}\right)} \phi(t) A_{\tilde{p}}(\mathbf{z}, t, \tau, \mu) A_{T R}\left(\omega_{0}, \mathbf{z}, t, \tau\right) .
$$

We refer to $\mathcal{F}$ as the forward modeling operator.

We use $\omega_{0}=2 \pi f_{0}$ and let $\tilde{f}_{d}=f_{0}(1-\mu)$. The phase of the forward operator becomes

$$
\varphi(t, \boldsymbol{z}, \tau, \mu)=2 \pi t\left[f_{d}(\tau, \mathbf{z})-f_{0}(1-\mu)\right]=2 \pi t\left(f_{d}(\tau, \mathbf{z})-\tilde{f}_{d}\right)
$$

where

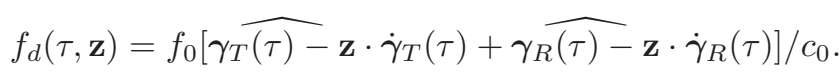

Note that $f_{d}$ is the bistatic Doppler frequency induced by the radial movements of the transmitter and receiver with respect to the scatterer $\boldsymbol{z}$ on the ground.

We assume that for some $m_{A}, A$ satisfies the inequality

$$
\sup _{(t, \mu, \tau, \boldsymbol{z}) \in \mathcal{U}}\left|\partial_{t}^{\alpha_{t}} \partial_{\mu}^{\alpha_{\mu}} \partial_{\tau}^{\beta} \partial_{z_{1}}^{\epsilon_{1}} \partial_{z_{2}}^{\epsilon_{2}} A(\boldsymbol{z}, t, \tau, \mu)\right| \leq C_{A}\left(1+t^{2}\right)^{\left(m_{A}-\left|\alpha_{t}\right|\right) / 2}
$$




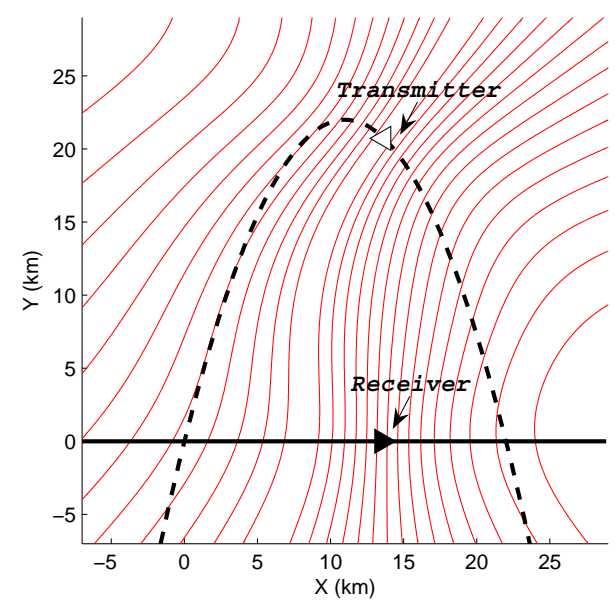

Figure 1: Iso-Doppler contours $F(\tau, \mu)$ for the bistatic Doppler frequency $f_{d}(0.5388 \mathrm{~s}, \mathbf{z})$. The transmitter is traversing a parabolic flight trajectory as indicated by the dashed line and the receiver is traversing a straight flight trajectory over a flat topography as indicated by the solid line (for the explicit form of the trajectories, see Section 4). The white and black triangles denote the position of the transmitter and receiver at 51.7241s and $52.2628 \mathrm{~s}$, respectively. The speed of the transmitter and receiver is $261 \mathrm{~m} / \mathrm{s}$.

where $\mathcal{U}$ is any compact subset of $\mathbb{R}^{+} \times \mathbb{R}^{+} \times \mathbb{R} \times \mathbb{R}^{2}$, and the constant $C_{A}$ depends on $\mathcal{U}, \alpha_{t, \mu}, \beta, \epsilon_{1,2}$. This assumption is needed in order to make various stationary phase calculations hold. In practice, (17) is satisfied for radar sufficiently away from the illuminated scene.

Under the assumption (17), (12) defines $\mathcal{F}$ as a Fourier integral operator whose leading-order contribution comes from those points lying in the intersection of the illuminated surface and points have the same bistatic Doppler frequency, i.e., $\left\{\mathbf{x} \in \mathbb{R}^{3}: f_{d}(\tau, \mathbf{z})=f_{0}(1-\mu)\right\}$. We denote the curves formed by this intersection by

$$
F(\tau, \mu)=\left\{\boldsymbol{z}: f_{d}(\tau, \mathbf{z})=f_{0}(1-\mu)\right\}
$$

and refer to $F(\tau, \mu)$ as an iso-Doppler contour. Figure 1 shows the iso-Doppler contours for the transmitter traversing a parabolic flight trajectory and the receiver traversing a straight flight trajectory over a flat topography.

\section{IMAGE FORMATION}

\subsection{Filtered Backprojection Operator}

Our objective is to reconstruct $\rho(\boldsymbol{z})$ based on the forward model given by (12). Since $\mathcal{F}$ is a Fourier integral operator, we form the image of $\rho(\boldsymbol{z})$ by a suitable filtered-backprojection of the data $d(\tau, \mu)$ onto $F(\tau, \mu)[10]$.

We form the image by filtered-backprojection as follows:

$$
\begin{aligned}
\tilde{\rho}(\boldsymbol{z}) & :=\mathcal{K}[d](\boldsymbol{z}) \\
& =\int \mathrm{e}^{\mathrm{i} \varphi(t, \boldsymbol{z}, \tau, \mu)} Q(\boldsymbol{z}, t, \tau) d(\tau, \mu) d t d \tau d \mu
\end{aligned}
$$

where $\mathcal{K}$ is referred to as the filtered-backprojection operator and $Q$ is the filter to be determined below. 


\subsection{Point Spread Function of the FBP Operator}

We rewrite $\tilde{\rho}(\boldsymbol{z})$ as

$$
\tilde{\rho}(\boldsymbol{z})=\int L\left(\boldsymbol{z}, \boldsymbol{z}^{\prime}\right) \rho\left(\boldsymbol{z}^{\prime}\right) d \boldsymbol{z}^{\prime}
$$

where $L\left(\boldsymbol{z}, \boldsymbol{z}^{\prime}\right)$ is the point spread function given by

$$
L\left(\boldsymbol{z}, \boldsymbol{z}^{\prime}\right)=\int \mathrm{e}^{\mathrm{i}\left[\varphi(t, \boldsymbol{z}, \tau, \mu)-\varphi\left(t^{\prime}, \boldsymbol{z}^{\prime}, \tau, \mu\right)\right]} Q(\boldsymbol{z}, t, \tau) A\left(\boldsymbol{z}^{\prime}, t^{\prime}, \tau, \mu\right) d t d t^{\prime} d \tau d \mu
$$

We define

$$
\Phi_{K}:=\varphi(t, \boldsymbol{z}, \tau, \mu)-\varphi\left(t^{\prime}, \boldsymbol{z}^{\prime}, \tau, \mu\right)
$$

and use the stationary phase theorem to approximate the $t^{\prime}$ and $\mu$ integrations of (21). We compute

$$
\partial_{t^{\prime}} \Phi_{K}=f_{d}\left(\tau, \boldsymbol{z}^{\prime}\right)-f_{0}(1-\mu)
$$

and

$$
\partial_{\mu} \Phi_{K}=2 \pi f_{0}\left(t-t^{\prime}\right) .
$$

The stationary points of the phase satisfying $\partial_{t^{\prime}, \mu} \Phi_{K}=0$ imply that

$$
\mu=1-f_{d}\left(\tau, \boldsymbol{z}^{\prime}\right) / f_{0}
$$

and $t=t^{\prime}$.

Substituting the results back into (21), we obtain

$$
L\left(\boldsymbol{z}, \boldsymbol{z}^{\prime}\right)=\int \mathrm{e}^{\mathrm{i} 2 \pi t\left[f_{d}(\tau, \boldsymbol{z})-f_{d}\left(\tau, \boldsymbol{z}^{\prime}\right)\right]} Q(\boldsymbol{z}, t, \tau) A\left(\boldsymbol{z}^{\prime}, t, \tau, 1-f_{d}\left(\tau, \boldsymbol{z}^{\prime}\right) / f_{0}\right) d t d \tau .
$$

For notational simplicity, we let

$$
\begin{aligned}
Q\left(\boldsymbol{z}, t, \tau, \boldsymbol{z}^{\prime}\right) & =Q\left(\boldsymbol{z}, t, \tau, 1-f_{d}\left(\tau, \boldsymbol{z}^{\prime}\right) / f_{0}\right) \\
A\left(\boldsymbol{z}^{\prime}, t, \tau\right) & =A\left(\boldsymbol{z}^{\prime}, t, \tau, 1-f_{d}\left(\tau, \boldsymbol{z}^{\prime}\right) / f_{0}\right)
\end{aligned}
$$

for the rest of our paper.

\subsection{Determination of the FBP Filter}

To determine the filter, we linearize $f_{d}\left(\tau, z^{\prime}\right)$ around $\boldsymbol{z}^{\prime}=\boldsymbol{z}$ using the fundamental theorem of calculus [11] and approximate

$$
f_{d}(\tau, \boldsymbol{z})-f_{d}\left(\tau, \boldsymbol{z}^{\prime}\right) \approx\left(\boldsymbol{z}-\boldsymbol{z}^{\prime}\right) \cdot \boldsymbol{\Xi}\left(\tau, \boldsymbol{z}, \boldsymbol{z}^{\prime}\right)
$$

and

$$
\begin{aligned}
Q\left(\boldsymbol{z}, t, \tau, \boldsymbol{z}^{\prime}\right) & =Q(\boldsymbol{z}, t, \tau, \boldsymbol{z}) \\
A\left(\boldsymbol{z}^{\prime}, t, \tau\right) & =A(\boldsymbol{z}, t, \tau)
\end{aligned}
$$

where in $(29)$

$$
\boldsymbol{\Xi}\left(\tau, \boldsymbol{z}, \boldsymbol{z}^{\prime}\right)=\int_{0}^{1} \nabla f_{d}\left(\tau, \boldsymbol{z}+\lambda\left(\boldsymbol{z}^{\prime}-\boldsymbol{z}\right)\right) d \lambda
$$

For notational simplicity, we let $Q(\boldsymbol{z}, t, \tau)=Q(\boldsymbol{z}, t, \tau, \boldsymbol{z})$ for the rest of our paper.

For each $\boldsymbol{z}$, we make the following change of variables:

$$
(t, \tau) \rightarrow \boldsymbol{\xi}=t \boldsymbol{\Xi}(\tau, \boldsymbol{z}, \boldsymbol{z})
$$


in the integral of (26) and then we have

$$
L\left(\boldsymbol{z}, \boldsymbol{z}^{\prime}\right)=\int_{\Omega_{\boldsymbol{z}}} \mathrm{e}^{\mathrm{i} 2 \pi\left(\boldsymbol{z}-\boldsymbol{z}^{\prime}\right) \cdot \boldsymbol{\xi}} Q(\boldsymbol{z}, \boldsymbol{\xi}) A(\boldsymbol{z}, \boldsymbol{\xi}) \eta(\boldsymbol{z}, \boldsymbol{\xi}) d \boldsymbol{\xi}
$$

where

$$
\begin{aligned}
& Q(\boldsymbol{z}, \boldsymbol{\xi})=Q(\boldsymbol{z}, t(\boldsymbol{\xi}), \tau(\boldsymbol{\xi})) \\
& A(\boldsymbol{z}, \boldsymbol{\xi})=A(\boldsymbol{z}, t(\boldsymbol{\xi}), \tau(\boldsymbol{\xi}))
\end{aligned}
$$

and

$$
\eta(\boldsymbol{z}, \boldsymbol{\xi})=\left|\frac{\partial(t, \tau)}{\partial \boldsymbol{\xi}}\right|=\left|\operatorname{det}\left[\begin{array}{c}
\boldsymbol{\Xi}(\tau, \boldsymbol{z}, \boldsymbol{z}) \\
\partial_{\tau} \boldsymbol{\Xi}(\tau, \boldsymbol{z}, \boldsymbol{z})
\end{array}\right]\right|^{-1}
$$

is the determinant of the Jacobian that comes from the change of variables given by (33). The notation det in (36) denotes matrix determinant. In (34),

$$
\Omega_{\boldsymbol{z}}=\left\{\boldsymbol{\xi}=t \boldsymbol{\Xi}(\tau, \boldsymbol{z}, \boldsymbol{z}) \mid A(\boldsymbol{z}, t, \tau) \neq 0,(t, \tau) \in\left(\mathbb{R}^{+}, \mathbb{R}\right)\right\} .
$$

We refer to $\Omega_{\boldsymbol{z}}$ as the data collection manifold at $\boldsymbol{z}$. This set determines many of the properties of the image.

Using (32) and (16), we obtain

$$
\boldsymbol{\Xi}(\tau, \boldsymbol{z}, \boldsymbol{z})=-\frac{f_{0}}{c_{0}} D \psi(\boldsymbol{z}) \cdot\left[\frac{\dot{\boldsymbol{\gamma}}_{T, \perp}(\tau, \boldsymbol{z})}{\left|\gamma_{T}(\tau)-\mathbf{z}\right|}+\frac{\dot{\boldsymbol{\gamma}}_{R, \perp}(\tau, \boldsymbol{z})}{\left|\gamma_{R}(\tau)-\mathbf{z}\right|}\right]
$$

where

$$
D \psi(\boldsymbol{z})=\left[\begin{array}{ccc}
1 & 0 & \partial \psi(\boldsymbol{z}) / \partial z_{1} \\
0 & 1 & \partial \psi(\boldsymbol{z}) / \partial z_{2}
\end{array}\right]
$$

and $\dot{\gamma}_{T(R), \perp}(\tau, \boldsymbol{z})$ is the projection of $\dot{\gamma}_{T(R)}(\tau)$ onto the plane normal to $\widehat{\gamma_{T(R)}(\tau)}-\mathbf{z}$ given by

$$
\dot{\gamma}_{T(R), \perp}(\tau, \mathbf{z})=\dot{\gamma}_{T(R)}(\tau)-\gamma_{T(R)}(\tau)-\mathbf{z}\left(\left(\gamma_{T(R)} \widehat{(\tau)}-\mathbf{z}\right) \cdot \dot{\gamma}_{T(R)}(\tau)\right) .
$$

We choose the filter as follows:

$$
Q(\boldsymbol{z}, t, \tau)=\chi_{\Omega_{\boldsymbol{z}}}(\boldsymbol{\xi}(t, \tau)) \frac{\overline{A(\boldsymbol{z}, t, \tau)}}{|A(\boldsymbol{z}, t, \tau)|^{2}} \frac{1}{\eta(\boldsymbol{z}, \boldsymbol{\xi})}
$$

where $\chi_{\Omega_{z}}$ is a smooth cut-off function equal to one in the interior of $\Omega_{z}$ and zero in the exterior of $\Omega_{z}$. Note that we choose the filter to make the point spread function $L\left(\boldsymbol{z}, \boldsymbol{z}^{\prime}\right)$ in (34) be the Dirac delta function.

Substituting (41) into (34) and the result back into (19), we obtain

$$
\begin{aligned}
\tilde{\rho}(\boldsymbol{z}) & :=\mathcal{K} \mathcal{F}[\rho](\boldsymbol{z}) \\
& =\int_{\Omega_{\boldsymbol{z}}} \mathrm{e}^{\mathrm{i} 2 \pi\left(\boldsymbol{z}-\boldsymbol{z}^{\prime}\right) \cdot \boldsymbol{\xi}} \rho\left(\boldsymbol{z}^{\prime}\right) d \boldsymbol{z}^{\prime} d \boldsymbol{\xi} .
\end{aligned}
$$

(42) shows that the image $\tilde{\rho}$ is a band-limited version of $\rho$ whose bandwidth is determined by the data collection manifold $\Omega_{\boldsymbol{z}}$, which describes the many of properties of the image. The larger the data collection manifold, the better the resolution of the reconstructed image is.

(42) shows that irrespective of the choice of the filter, the backprojection operator recovers the visible edges of the scene at the correct location. With the choice of filter given in (41), the resulting image formation algorithm can recover not only the correct location and orientation, but also the correct strength of the visible edges [12-15]. 


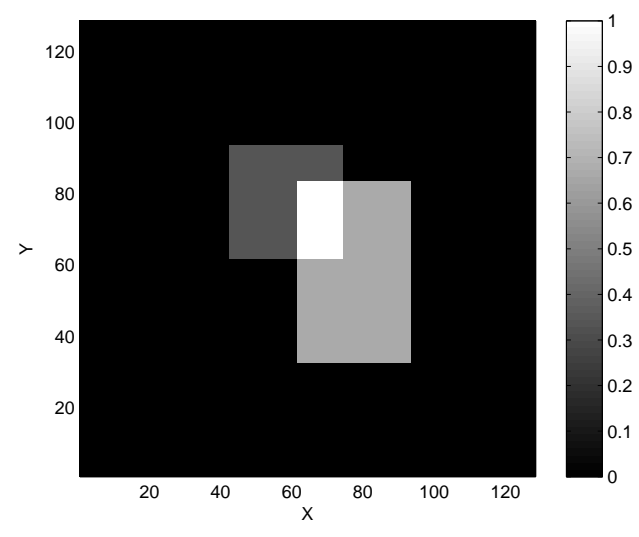

Figure 2: Scene with extended targets used in the numerical simulations.

\section{NUMERICAL SIMULATIONS}

\subsection{Settings}

We considered a scene of size $[0,11 \mathrm{e} 3] \times[0,11 \mathrm{e} 3] \mathrm{m}^{2}$ with flat topography centered at $[11,11,0] \mathrm{km}$. The scene was discretized by $128 \times 128$ pixels, where $[0,0,0] \mathrm{m}$ and $[11 \mathrm{e} 3,11 \mathrm{e} 3,0] \mathrm{m}$ corresponds to the pixels $(1,1)$ and $(128,128)$, respectively.

We assumed that there are two partially overlapped extended targets present in the scene, as shown in Fig. 2. One is a square target and the other is a rectangular target.

We considered the imaging with two different flight trajectory set-ups. In the first case, the transmitter was assumed to be traversing a parabolic trajectory,

$$
\gamma_{T}(s)=\left[s,\left(22 s-s^{2}\right) * 22 / 121,6.5\right] \mathrm{km}
$$

and the receiver was assumed to be traversing a linear trajectory,

$$
\gamma_{R}(s)=(s, 0,6.5) \mathrm{km} .
$$

Note that the variable $s$ in $\gamma_{T}$ and $\gamma_{R}$ is equal to $V t$ where $V$ is the speed of the receiver. In the second case, both the transmitter and the receiver were traversing a circular trajectory given by

$$
\gamma_{C}(s)=(11+11 \cos (s), 11+11 \sin (s), 6.5) \mathrm{km} \text {. }
$$

Let $\gamma_{T}(s)$ and $\gamma_{R}(s)$ denote the trajectories of the transmitter and receiver. We set $\gamma_{T}(s)=\gamma_{C}(s)$ and $\gamma_{R}(s)=$ $\gamma_{C}\left(s-\frac{\pi}{4}\right)$. Note that the variable $s$ in $\gamma_{C}$ is equal to $\frac{V}{R} t$, where $V$ is the speed of the receiver, and $R$ is the radius of the circular trajectory. In both cases above, we set the speed of the transmitter and receiver to $261 \mathrm{~m} / \mathrm{s}$.

Fig. 3 shows $2 \mathrm{D}$ view of the scene with the targets, and the trajectories of the transmitter and receiver in the two cases above.

For all the experiments, we assumed that the transmitter transmited a single-frequency continuous waveform operating at $f_{0}=\omega_{0} / 2 \pi=800 \mathrm{MHz}$, i.e., $\tilde{p}(t)$ in (2) was set to be unit. We used (2) to generate the data and chose the windowing function $\phi$ in (5) to be a Hanning function.

\subsection{Results}

The reconstructed image with two different flight trajectory set-ups are shown in Fig. 4(a) and Fig. 4(b), respectively. We reconstructed image with the length of the signal $L_{\phi}=0.0427 \mathrm{~s}$ and the sampling rate of the aperture $f_{\tau}=151.8545 \mathrm{~Hz}$. We can see the two extended targets are reconstructed successfully in both cases.

Comparing Fig. 4(b) and Fig. 4(a), the reconstructed image in Fig. 4(b) is better than that in Fig. 4(a). This is due to the full aperture exploited in the case of circular trajectory, which corresponds to a larger data collection manifold and therefore leads to a better image quality. 


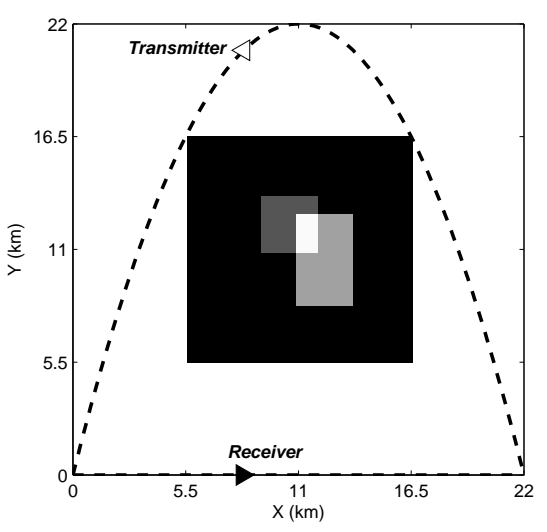

(a)

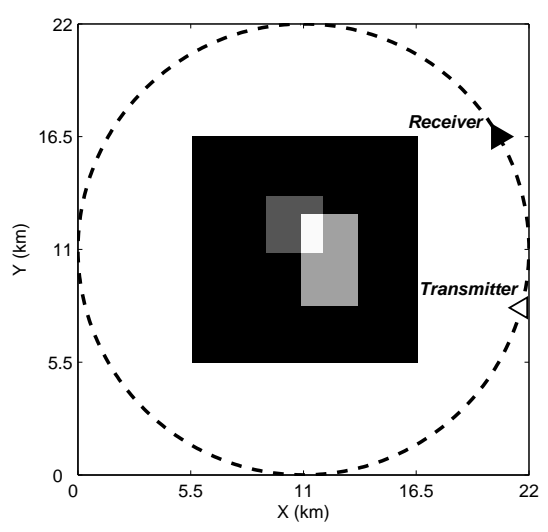

(b)

Figure 3: The 2-D view of the simulation set-ups. (a) The transmitter is traversing a parabolic trajectory shown by the dashed line. The receiver is traversing a linear trajectory shown by the bold solid line; (b) Both the transmitter and the receiver are traversing a circular trajectory shown by the dashed line. In (a) and (b), the dark region denotes the scene considered in the simulations. At a certain time instant, the transmitter and the receiver are located at the positions shown in the figure. For the explicit form of the trajectories, see (43), (44) and (45).

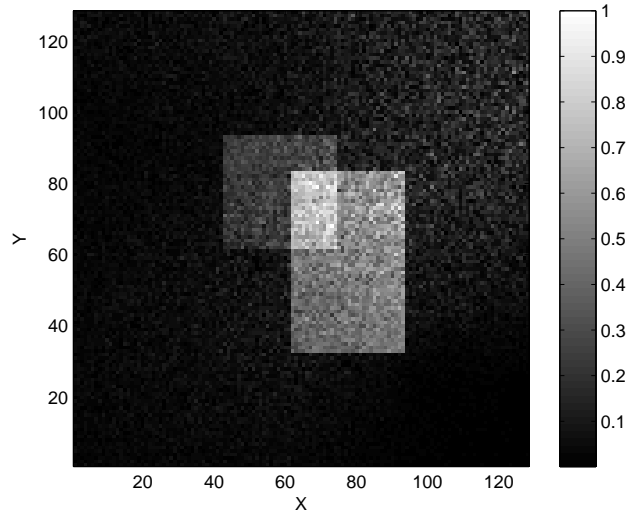

(a)

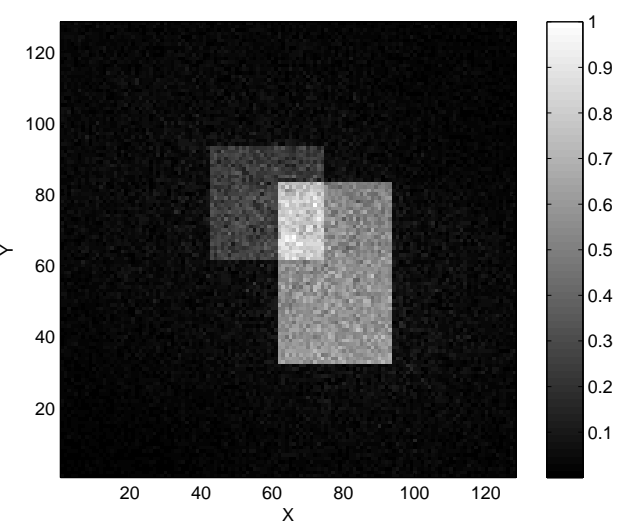

(b)

Figure 4: (a)The reconstructed image with the transmitter and receiver traversing a parabolic flight trajectory $\gamma_{T}(s)$ and a linear flight trajectory $\gamma_{R}(s)$, respectively, as shown in (43) and (44), and Fig. 3(a). (b)The reconstructed image with the transmitter and receiver traversing a circular flight trajectory $\gamma_{C}(s)$ as shown in (45) and Fig. 3(b), where $\gamma_{T}(s)=\gamma_{C}(s)$ and $\gamma_{T}(s)=\gamma_{C}(s-\pi / 4)$, respectively. In (b) and (c), The transmitter was assumed to be transmitting a single-frequency waveform at $f_{0}=800 \mathrm{MHz}$. The length of the support of the windowing function is $L_{\phi}=0.0427 \mathrm{~s}$. The sampling rate of $\tau$ is $f_{\tau}=151.8545 \mathrm{~Hz}$. 


\section{CONCLUSION}

In this paper, we developed a novel image formation method for SAR that uses ultranarrow-band transmitted waveforms where the transmitter and receiver may traverse arbitrary trajectories. This method is based on the windowed, scaled and translated correlation of the received signal and transmitted waveform and the filtered backprojection of the resulting correlated signal on to the bistatic iso-Doppler curves. The numerical simulations demonstrate the performance of the reconstruction.

\section{REFERENCES}

1. Mensa, D., Halevy, S., and Wade, G., "Coherent doppler tomography for microwave imaging," Proceedings of the IEEE 71, 254-261 (Feb. 1983).

2. Mensa, D. and Heidbreder, G., "Bistatic synthetic-aperture radar imaging of rotating objects," IEEE Transactions on Aerospace and Electronic Systems 18, 423-431 (July 1982).

3. Sun, H., Feng, H., and Lu, Y., "High resolution radar tomographic imaging using single-tone cw signals," in [2010 IEEE Radar Conference], 975-980 (May 2010).

4. Wicks, M. C., Himed, B., Bracken, J. L. E., Bascom, H., and Clancy, J., "Ultra narrow band adaptive tomographic radar," in [IEEE International workshop on computational advances in multi-sensor adaptive processing], 36-39 (Dec. 2005).

5. Coetzee, S. L., Baker, C. J., and Griffiths, H. D., "Narrow band high resolution radar imaging," in [2006 IEEE Radar Conference], 622-625 (April 2006).

6. Borden, B. and Cheney, M., "Synthetic-aperture imaging from high-doppler-resolution measurements," Inverse Problems 21, 1-11 (Nov. 2005).

7. Thomson, J. H. and Ponsonby, J. E. B., "Two-dimensional aperture synthesis in lunar radar astronomy," Proc. R. Soc. London Ser. A 303 , 477-491 (1968).

8. Roulston, M. S. and Muhleman, D. O., "Synthesizing radar maps of polar regions with a doppler-only method," Applied Optics 36, 3912-3919 (June 1997).

9. Yarman, C. E., Wang, L., and Yazıcı, B., "Doppler synthetic aperture hitchhiker imaging," Inverse Problems 26 (065006(26pp) 2010).

10. Treves, F., [Introduction to Pseudodifferential and Fourier Integral Operators, volumes I and II], Plenum Press, New York (1980).

11. Courant, R. and John, F., [Introduction to Calculus and analysis, vol. 1 and 2], Springer, New York (2000).

12. Nolan, C. J. and Cheney, M., "Synthetic aperture inversion," Inverse Problems 18, 221-236 (2002).

13. Nolan, C. J. and Cheney, M., "Microlocal analysis of synthetic aperture radar imaging," The Journal of Fourier Analysis and Applications 10, 133-148 (2004).

14. Yazici, B., Cheney, M., and Yarman, C. E., "Synthetic-aperture inversion in the presence of noise and clutter," Inverse Problems 22, 1705-1729 (2006).

15. Yarman, C. E., Yazıcı, B., and Cheney, M., "Bistatic synthetic aperture radar imaging for arbitrary flight trajectories," Image Processing, IEEE Transactions on 17, 84-93 (Jan. 2008). 